\title{
Results of Curative Radiation Therapy with or without Chemotherapy for Stage III Unresectable Non-Small Cell Lung Cancer
}

\author{
Sung-Ja Ahn, M.D. ${ }^{1}$, Young-Chul Kim, M.D. ${ }^{2}$, Kyu-Sik Kim, M.D. ${ }^{2}$, Kyung-Ok Park, M.D. ${ }^{3}$, Woong-Ki \\ Chung, M.D. ${ }^{1}$, Taek-Keun Nam, M.D. ${ }^{1}$, Byung-Sik Nah, M.D. ${ }^{1}$, Ju-Young Song, Ph.D. ${ }^{1}$ and Mi-Sun Yoon, \\ M.D. ${ }^{1}$
}

Departments of ${ }^{1}$ Radiation Oncology, ${ }^{2}$ Internal Medicine, Chonnam National University Medical School, ${ }^{3}$ Department of Internal Medicine, Seonam University Medical School, Gwangju, Korea

Purpose: We retrospectively analyzed the patients who received curative radiotherapy for unresectable stage III NSCLC to investigate the impact of chemotherapy.

Materials and Methods: From 1998 to 2001, the records of 224 patients who completed curative radiotherapy for NSCLC were reviewed. There were 210 males and 14 females, and their median age was 64 years (range 38 23). 54 patients had stage IIIA disease and 170 patients had stage IIIB disease. Conventional radiotherapy was given and the radiation dose ranged from 50 70 Gy with a median of $60 \mathrm{~Gy}$, and chemotherapy was combined for 116 patients $(52 \%)$.

Results: The median survival, the 2-year, and 5-year actuarial survival rates of all 224 patients were 15 months, $30 \%$, and $7 \%$, respectively. The median survival of the patients with stage IIIA and IIIB disease were 21 months and 13 months, respectively $(p=0.14)$. The median survival of patients who received chemoradiation was 18 months compared to 14 months for the patients who received $R T$ alone $(p=0.02)$. Among the chemoradiation group of patients, the median survival time of the patients who received 1 to 3 cycles of chemotherapy was 16 months and that for the patients who received more than 3 cycles was 22 months $(p=0.07)$. We evaluated the effects of the timing of chemoradiation in 57 patients who received more than 3 cycles of chemotherapy. The median survival of the patients with the concurrent sequence was 25 months and that for the patients with the sequential chemotherapy was 19 months $(p=0.81)$.

Conclusions: For advanced stage III non-small cell lung cancer patients who completed the curative radiotherapy, the addition of chemotherapy improved the survival compared to the patients who received radiotherapy alone. (Cancer Res Treat. 2005;37:268-272)

Key words: Radiotherapy, Chemotherapy, Non-small cell lung cancer

\section{INTRODUCTION}

Lung cancer currently represents the leading cause of death from malignant disease in Korea as well as in Western countries. Non-small-cell lung cancer (NSCLC) constitutes approximately three-quarters of the primary malignant lung tumors and it is unresectable in $70 \%$ of the cases with approximately $40 \%$ of the patients having stage III disease. Because of the limited benefits provided by radiation therapy (RT) alone, investigators have examined a variety of modified and combined treatment modalities, ranging from altered

Correspondence: Sung-Ja Ahn, Department of Radiation Oncology, Chonnam University Medical School, 8 Hak-dong, Dong-gu, Gwangju 501-757, Korea. (Tel) 82-61-379-7200, (Fax) 82-61-379-7249,

(E-mail) ahnsja@chonnam.ac.kr

Received August 8, 2005, Accepted September 27, 2005 fractionation to various combinations of chemotherapy (ChT). Combined chemoradiation appears to have improved the outcome of patients with locally advanced, unresectable stage III NSCLC, and these patients have a median survival of 13 to 14 months and 5-year survival rates as high as $15 \%$ to $20 \%$, which is nearly three times that reported with RT alone. In 1998, Choy et al reported the results of a multiinstitutional phase II clinical trial and they showed that a combined modality therapy with paclitaxel, carboplatin and radiation is a promising treatment for locally advanced NSCLC with a high response rate and acceptable toxicity and survival rates (1). Based on the evidence in the combined-modality arm of that study showing a statistically significant survival benefit, we have followed, since 1998, the treatment policy of a combined modality therapy for patients with unresectable stage III NSCLC. By performing this analysis, we wanted to elucidate the role of chemotherapy for the management of patients with unresectable stage III non-small cell lung cancer. 


\section{MATERIALS AND METHODS}

From 1998 and 2001, we retrospectively reviewed the records of 224 patients who had completed the curative radiotherapy with or without chemotherapy for NSCLC. There were 210 male $(93.8 \%)$ and 14 female $(6.2 \%)$ patients, and their median age was 64 years (range: $38 \sim 83$ years). 165 (73.7\%) patients had squamous cell carcinoma and 40 patients (17.8\%) had adenocarcinoma; 54 patients $(24.1 \%)$ had stage IIIA disease and 170 patients $(75.9 \%)$ had stage IIIB disease.

All the patients underwent evaluations that included a physical examination, complete blood chemistry, standard chest x-rays, computed tomography (CT) scans of the chest and upper abdomen, bronchoscopy with detailed anatomic mapping of the endobronchial lesions and pulmonary function testing. ${ }^{99 \mathrm{~m}}$ Tc-whole body bone scans and brain CT or MRI were performed when clinically indicated. The patients underwent bronchoscopic or transthoracic needle aspiration biopsy for the histologic diagnosis. Blood chemistry and sputum examinations for tuberculosis were routinely done for all the patients. Stage assignment was performed using the AJCC/UICC system (2001), as based on the clinical information.

Conventional radiotherapy was delivered with $6 \mathrm{MV}$ (Mevatron, Siemens Co., Germany) or 10 MV (Varian Co., Palo alto, CA) X-rays. The patients were immobilized in a Vac-Lock (Meditec, Orange, IA) with both arms elevated above head. Simulation was performed using a CT-Simulator (MHTI, Miami, FL) in the treatment position using a $10 \mathrm{~mm}$ slice interval. Initially we used the parallel opposing AP-PA fields to $40 \mathrm{~Gy}$. The target volume consisted of the primary tumor, the nodal metastasis and the first uninvolved nodal chain. We changed the treatment plan one to three times to boost the gross tumor volume in addition to limit the critical normal tissue dose within the tolerance. The fraction size of $1.8,2.0$ or $2.5 \mathrm{~Gy}$ was given 5 times a week and this was prescribed to the intersection of the central axis of the treatment fields. No lung correction was used in the dose calculations. The planned dose to the gross tumor volume was usually 60 Gy (range: $50 \sim 70$ Gy, median: 60 Gy).

Chemotherapy was combined in 116 patients (52\%). Of those patients, 48 patients (41\%) had neoadjuvant ChT, 45 (39\%) had concurrent ChT and 23 (20\%) had post-radiation adjuvant ChT. Cis-platinum was combined with various drugs, i.e., with Taxol $(\mathrm{N}=64)$, with Gemzar or Navelbine $(\mathrm{N}=10)$, with Etoposide $(\mathrm{N}=20)$, and with Methotrexate+Vinblastine $(\mathrm{N}=3)$. The course of chemotherapy ranged from 1 to 10 with a median of 4 cycles ( $1 \sim 3$ in 54 patients, $4 \sim 6$ in 54 patients and $7 \sim 10$ in 3 patients).

The survival status was identified in 219 patients (98\%) via the medical records or by mail. The survival time was calculated from the first date of treatment to the date of death or to the date of the last follow-up. The median follow-up time of all the patients was 27 months with the range of 3 76 months. The SPSS software system (SPSS for Windows, version 11.0; SPSS Inc, Chicago, IL) was used for the statistical analysis. The survival curves were plotted using the KaplanMeier method, and the statistical significance was assessed using the log-rank test. A Cox proportional hazards regression model was used to identify the statistically significant differences in survival.

\section{RESULTS}

The median survival, the 2-year, 3-year and 5-year actuarial survival rates of all 224 patients were 15 months, 30\%, 19\% and $7 \%$, respectively (Fig. 1). We then compared the survival times according to the clinical variables (Table 1). The median survival times of the patients with stage IIIA and IIIB disease were 21 months and 13 months, respectively $(p=0.14)$. The median survival of the patients with a radiation dose lower than or above 61 Gy were 15 months and 18 months, respectively $(p=0.44)$. The median survival times of the patients who received chemotherapy and RT alone were 18 months and 14 months, respectively $(\mathrm{p}=0.02)$. There was no statistically significant prognostic factor in the multivariate analysis among these variables (Table 1).

The clinical variables between the RT alone group and the chemoradiation group showed no statistically significant difference (Table 2). The median survival time of the patients who received 1 to 3 cycles of chemotherapy $(\mathrm{N}=59)$ was 16 months and the median survival time of the patients who received over 3 cycles $(\mathrm{N}=57)$ was 22 months $(\mathrm{p}=0.07)$. We evaluated the effect of the timing of chemoradiation in the 57 patients who had received more than 3 cycles of chemotherapy (Fig. 2). The median survival time of the patients who received concurrent ChT $(\mathrm{N}=20)$ was 25 months and that for the patients who received neoadjuvant ChT $(\mathrm{N}=24)$ was 19 months, and that for the patients who received post-radiation adjuvant $\mathrm{ChT}(\mathrm{N}=13)$ was 17 months $(p=0.81)$. On the multivariate analysis comparing the RT alone group and the chemoradiation group who received more than 3 cycles of chemotherapy, the combination of ChT was the most powerful prognostic factor in the survival analysis (Table 3).

The overall failure rate was $87 \%$ and the median time to the treatment failure was 9 months. The local and distant failure

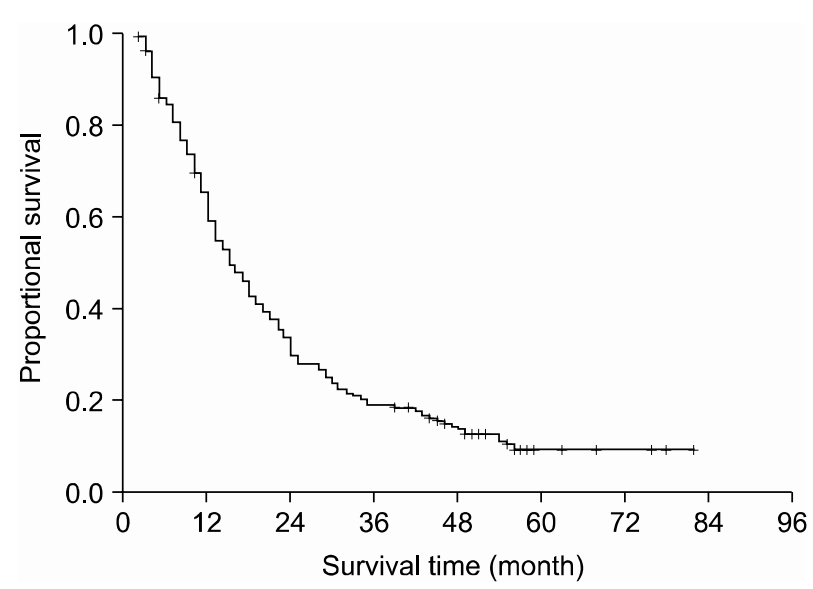

Fig. 1. The overall survival of all 224 patients with stage III non-small-cell lung cancer. 
Table 1. Survival analysis according to the clinical variables in all the patients $(N=224)$

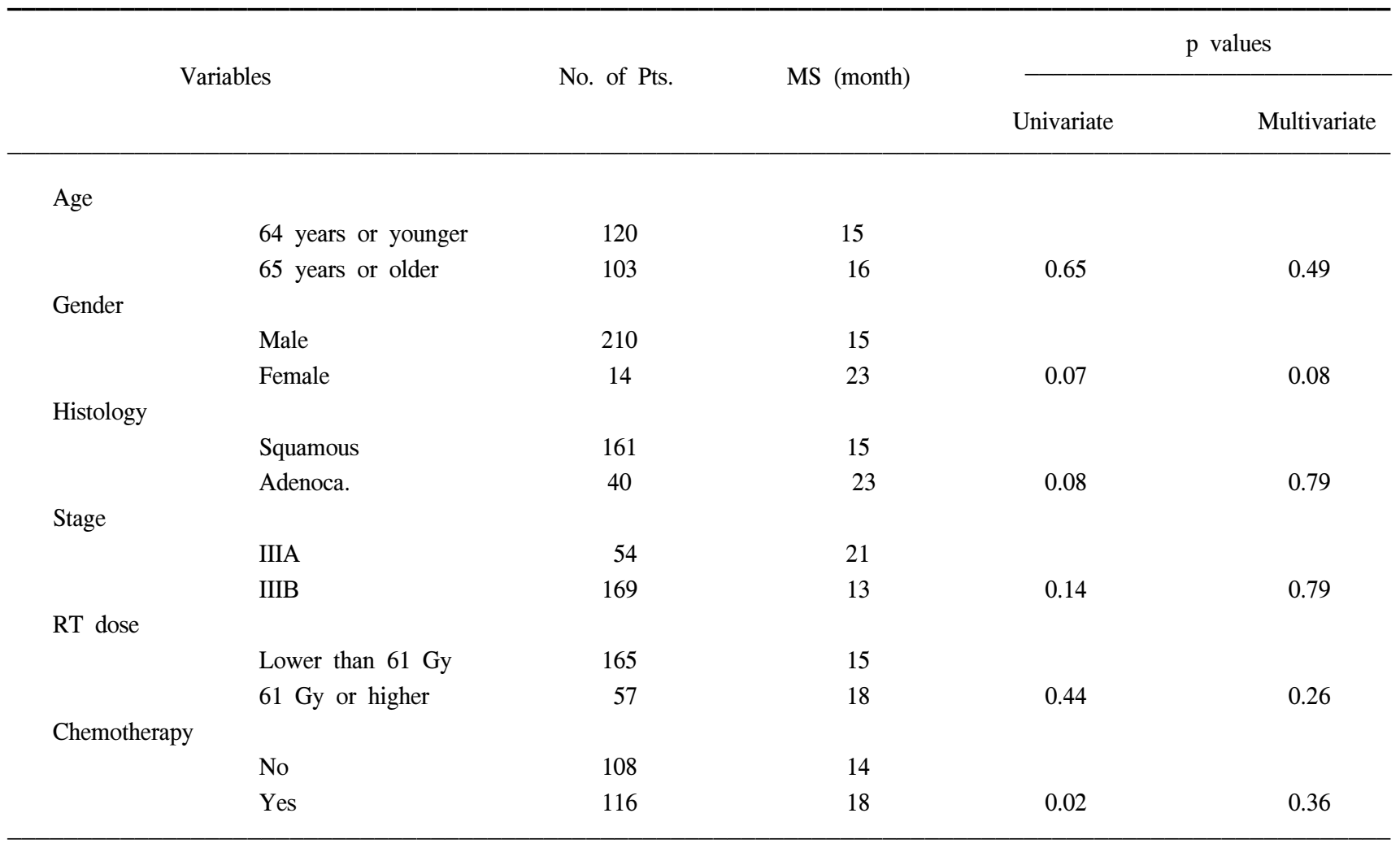

Table 2. Comparison of the clinical variables in the patient group according to the treatment modality

\begin{tabular}{cccc}
\hline Variables & $\begin{array}{c}\text { RT alone } \\
(\mathrm{N}=108)\end{array}$ & $\begin{array}{c}\text { Chemoradiation } \\
(\mathrm{N}=116)\end{array}$ & $\mathrm{p}$ values \\
\hline Stage & & & \\
IIIA & 27 & 27 & 0.76 \\
IIIB & 81 & 89 & \\
PS Score & & & \\
0 & 5 & 78 & 0.69 \\
1 & 82 & 26 & \\
2 & 21 & 101 & 0.88 \\
WT. Loss & & 15 & \\
$<10 \%$ & 93 & & 0.24 \\
$>10 \%$ & 15 & $54 \sim 70$ & \\
RT Dose & & 60 & \\
Range & $50 \sim 70$ & & \\
median & 60 & & \\
\hline
\end{tabular}

rates were $79 \%$ and $52 \%$, respectively. The median times to local and distant failures were 14 and 56 months, respectively. Local failure without evidence of distant metastasis was shown in $69 \%$ of the patients $(120 / 224)$, which can be compared to

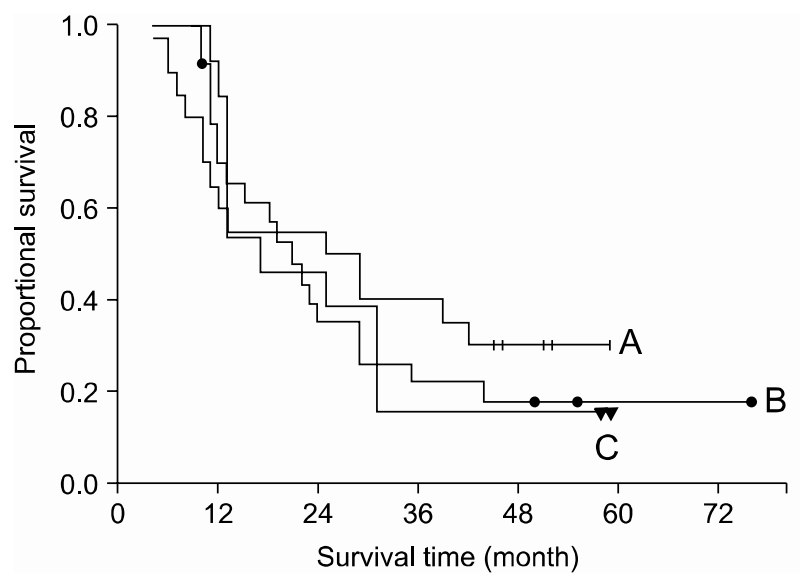

Fig. 2. The survival curve according to the timing of chemoradiation was shown in the patient group who received chemotherapy more than 3 cycles. (A) concurrent $(\mathrm{N}=20)$, (B) neoadjuvant $(\mathrm{N}=24),(\mathrm{C})$ post-radiation group $(\mathrm{N}=13)$.

a distant metastasis-only rate of $18 \%$ (32/224). The local failure within the radiation port was predominant and failure outside the radiation port occurred in only 10 patients $(7 \%)$. The pattern of failures was compared according to the treatment modality (Table 4). There was an even distribution of the failure patterns without any statistically significant difference between the two groups. 
Table 3. Multivariate analysis of the prognostic factors affecting to the overall survival

\begin{tabular}{lc}
\hline \multicolumn{1}{c}{ Parameters } & p-value \\
\hline Chemotherapy & 0.01 \\
Histology & 0.02 \\
Gender & 0.09 \\
Age_64 yrs & 0.56 \\
Stage & 0.40 \\
Radiation dose & 0.34 \\
\hline
\end{tabular}

Table 4. Patterns of failures according to the treatment modality

\begin{tabular}{llcc}
\hline Patterns & $\begin{array}{c}\text { All (\%) } \\
(\mathrm{N}=108)\end{array}$ & $\begin{array}{c}\text { RT alone } \\
(\mathrm{N}=116)\end{array}$ & Chemoradiation \\
\hline Local & $120(69)$ & 64 & 56 \\
Local+Distant & $22(13)$ & 6 & 16 \\
In-field only & 128 & 64 & 64 \\
In+out-field & 3 & 2 & 1 \\
Out-field only & 10 & 3 & 7 \\
Distant & $32(18)$ & 17 & 15 \\
Brain & 18 & 7 & 11 \\
Bone & 18 & 6 & 12 \\
Lung & 14 & 8 & 6 \\
Liver & 8 & 2 & 6 \\
\hline
\end{tabular}

There was one case of RTOG grade 3 radiation esophagitis. Radiation pneumonitis (RTOG $\geqq G 3$ ) was not found in this analysis.

\section{DISCUSSION}

A number of randomized clinical trials and meta-analyses have supported the conclusion that the combined modality approaches with using cisplatin-based chemotherapy improve patient survival compared with radiotherapy alone for the patients with surgically unresectable stage III NSCLC $(2 \sim 5)$. Our study also revealed the importance of a combination of chemotherapy in the management of such patient group. Depending on the strategy that is used, chemotherapy may play a cytotoxic role by eradicating distant micrometastases, a radiosensitizing role by improving local control, or both. Clearly, the interaction is multifactorial, and the dominant mechanism may be affected by specifics of the treatment settings that include the drug exposure and concentration, the tumor type and the radiation dosimetry.

Two randomized trials have directly compared the sequential and concurrent chemoradiation regimens. Each trial has demonstrated superior survival with the concurrent approach $(6,7)$.
Concurrent chemoradiation therapy yields better results than sequential therapy in terms of the time to progression and the increased survival. Concurrent chemoradiation has become the new standard treatment for unresectable stage III NSCLC (8). In our patients group who received more than 3 cycles of chemotherapy, the concurrent group showed a better median survival of 25 months as compared to 19 months of the sequential group, but this was without statistical significance. Although patients have substantially benefited from chemoradiotherapy, the 5-year survival rates are still very low. Poor local control is a major obstacle for achieving long-term cure in the patients with locally advanced NSCLC, as the 2-year in-field progression rates range from $26 \%$ to $45 \%$ after curative chemoradiotherapy $(9,10)$. There have been clinical trials evaluating the effect of consolidation chemotherapy with paclitaxel and carboplatin after concurrent chemoradiation for patients with stage III unresectable NSCLC $(11,12)$. They reported that consolidation chemotherapy was feasible and well tolerated. In our analysis of the survival difference by the number of chemotherapy cycles, we observed that improved survival paralleled to the increased number of cycles of chemotherapy. So, we would recommend the full cycles of consolidation chemotherapy at maximum of 4 after the initial concurrent chemoradiation if the general condition of the patient will permit it. For the aspect of radiation technique, three-dimensional conformal radiation therapy (3D-CRT) made it possible to increase the delivered dose of radiation without compromising the complications. The administration of higher doses using 3D-CRT improves the local control in stage III NSCLC patients and this can be led to improved survival (13, 14). RTOG launched a clinical trial of concurrent chemoradiation using 3D-CRT (L-0117) on 2001, and we are anticipating further improved treatment outcomes from this trial.

However, we still need to improve the local control and we have considered a trimodality therapy that includes surgery for the definitive local control of those patients with advanced disease $(15 \sim 17)$. Patients with locally advanced NSCLC have often been excluded from the treatment strategies that included surgery. Trodella et al (16) have evaluated the efficacy of induction chemoradiation and the role of surgical resection, when feasible, after induction therapy for locally advanced stage III NSCLC. The patients downstaged to stage 0-1 showed a statistically significant improved survival time (median: 32.5 months of the patients with $0-1$ stage disease versus 18.3 months for the patients with II-III stage disease; $p=0.025$ ). They have found that downstaging is directly and significantly correlated with the disease-free survival and the distant recurrence rate, and the rate of downstaging seems better for the neoadjuvant combined chemoradiation-treated patients than for the patients who were treated chemotherapy and radiotherapy as the only treatment. This underlines the impact of achieving local control on metastasis and survival. Based on these data, we have recently tried a trimodality treatment that surgical resection, when feasible, can be included by re-staging with PET-CT after concurrent chemoradiation in the patients with initially unresectable NSCLC. If the surgical resection cannot be done or if the patient refuses the surgery, we continue with the consolidation chemotherapy. We are awaiting the results of this treatment policy. 


\section{CONCLUSIONS}

For the advanced stage III non-small cell lung cancer patients who have a good performance status, the combination of chemotherapy improved the survival compared to using radiotherapy alone. However, our study had the limitation of a retrospective analysis for defining the most optimal schedule and dose of chemoradiation in the advanced stage NSCLC patient group.

\section{REFERENCES}

1. Choy H, Akerley W, Safran H, Graziano S, Chung C, Williams $\mathrm{T}$, et al. Multiinstitutional phase II trial of paclitaxel, carboplatin, a concurrent radiation therapy for locally advanced non-small-cell lung cancer. J Clin Oncol. 1998;16:3316-22.

2. Dillman RO, Herndon J, Seagren SL, Eaton WL Jr, Green MR. Improved survival in stage III NSCLC: Seven year follow-up of CALGB 8433 trial. J Natl Cancer Inst 1996;88:1210-15.

3. Le Chevalier T, Arriagada R, Quoix E, Ruffie P, Martin M, Tarayre M, et al. Radiotherapy alone versus combined chemotherapy and radiotherapy in non-resectable non-small cell lung cancer: First analysis of a randomized trial of 353 patients. J Natl Cancer Inst. 1991;83:417-23.

4. Non-Small Cell Lung Cancer Collaborative Group: Chemotherapy in non-small cell lung cancer: a meta-analysis using updated data on individual patients from 52 randomized clinical trials. Br Med J. 1995;311:899-909.

5. Sause W, Kolesar P, Taylor S IV, Johnson D, Livingston R, Komaki R, et al. Final results of phase III trial in regionally advanced unresectable non-small cell lung cancer: Radiation Therapy Oncology Group, Eastern Cooperative Oncology Group, and Southwest Oncology Group. Chest. 2000;117:35864.

6. Furuse K, Fukuoka M, Kawahara M, Nishikawa H, Takada Y, Kudoh S, et al. Phase III study of concurrent versus sequential thoracic radiotherapy in combination with mitomycin, vindesine, and cisplatin in unresectable stage III non-small-cell lung cancer. J Clin Oncol. 1999; 17:2692-99.

7. Curran W, Scott C, Langer R, Komaki R, Lee JS, Movsas B, et al. Phase III comparison of sequential vs concurrent chemoradiation for patients with unresected stage III non-small cell lung cancer (NSCLC): Report of Radiation Therapy Oncology Group (RTOG) 9410. Lung Cancer. 2000;29:93(abstr 303).

8. Gaspar LE. Optimizing chemoradiation therapy approaches to unresectable stage III non-small cell lung cancer. Curr Opin Oncol. 2001;13:110-5.

9. Komaki R, Seiferheld W, Ettinger D, Lee JS, Movsas B, Sause W. Randomized phase II chemotherapy and radiotherapy trial for patients with locally advanced inoperable non-small-cell lung cancer: long-term follow- up of RTOG 92-04. Int J Radiat Oncol Biol Phys. 2002;53:548-57.

10. Arriagada R. Current strategies for radiation therapy in nonsmall cell lung cancer. Chest. 1997;112(Suppl 4):S209-S13.

11. Kaplan B, Altynbas M, Eroglu C, Karahacioglu E, Er O, Ozkan $\mathrm{M}$, et al. Preliminary results of a phase II study of weekly paclitaxel (PTX) and carboplatin (CBDCA) administered concurrently with thoracic radiation therapy (TRT) followed by consolidation chemotherapy with PTX/CBDCA for stage III unresectable non-small-cell lung cancer (NSCLC). Am J Clin Oncol. 2004;27;6:603-10.

12. Gandara DR, Chansky K, Albain KS, Leigh BR, Gaspar LE, Lara PN, et al. Consolidation docetaxel after concurrent chemoradiotherpy in stage IIIB non-small- cell lung cancer: phase II Southwest Oncology Group Study S9504. J Clin Oncol. 2003;21:2004-10.

13. Bradley J, Graham MV, Winter K, Purdy JA, Komaki R, Roa WH, et al. Toxicity and outcome results of RTOG 9311: a phase I-II dose escalation study using three-dimensional conformal radiotherapy in patients with inoperable non-smallcell lung carcinoma. Int J Radiat Oncol Biol Phys. 2005;61: 318-28.

14. Rengan R, Rosenzweig KE, Venkatraman E, Koutcher LA, Fox JL, Nayak R, et al. Improved local control with higher doses of radiation in large-volume stage III non-small-cell lung cancer. Int J Radiat Oncol Biol Phys. 2004;60:741-7.

15. De Candis D, Stani SC, Bidoli P, Bedini VA, Potepan P, Navarria $P$, et al. Induction chemotherapy with carboplatin/ paclitaxel followed by surgery or standard radiotherapy and concurrent daily low-dose cisplatin for locally advanced nonsmall cell lung cancer (NSCLC). Am J Clin Oncol. 2003;26: 265-9.

16. Trodella L, Granone P, Valente S, Margaritora S, Macis G, Cesario A, et al. Neoadjuvant concurrent radiochemotherapy in locally advanced (IIIA-IIIB) non-small-cell lung cancer: longterm results according to downstaging. Ann Oncol. 2004;15: $389-98$.

17. Albain KS, Rusch VW, Crowley JJ, Rice TW, Turrisi AT, Weick JK, et al. Concurrent cisplatin/etoposide plus chest radiotherapy followed by surgery for stage IIIA(N2) and IIIB non-small-cell lung cancer: mature results of Southwest Oncology Group phase II study 8805. J Clin Oncol. 1995;13: 1880-92. 